\title{
SiRNA Directed Against Annexin II Receptor Inhibits Angiogenesis via Suppressing MMP2 and MMP9 Expression
}

\author{
Hongyuan Song ${ }^{\mathrm{a}}$ Dongyan Pan ${ }^{\mathrm{a}}$ Weifeng Sun ${ }^{\mathrm{a}}$ Cao Gu ${ }^{\mathrm{a}}$ Yuelu Zhang ${ }^{\mathrm{a}}$ \\ Ping Zhao ${ }^{b}$ Zhongtian $\mathrm{Q}^{\mathrm{b}}$ Shihong Zhao ${ }^{\mathrm{a}}$ \\ a'Department of Ophthalmology, Changhai Hospital, Second Military University, ${ }^{b}$ Department of \\ Microbiology, Shanghai Key Laboratory of Medical Biodefense, Second Military University, Shanghai, \\ China
}

\section{Key Words}

Annexin II receptor $•$ C5orf39 $•$ Angiogenesis $•$ Matrix metalloproteinase $\bullet$ siRNA

\begin{abstract}
Background/Aims: Annexin II receptor (AXIIR) is able to mediate Annexin II signal and induce apoptosis, but its role in angiogenesis remains unclear. This study tries to investigate the role of AXIR in angiogenesis and the plausible molecular mechanism. Methods/Results: RNA interference technology was used to silence AXIR, and the subsequent effects in vitro and in vivo were evaluated thereafter. Our data indicated that human umbilical vein endothelial cells (HUVECs) expressed AXIR and knockdown of AXIR significantly inhibited HUVECs proliferation, adhesion, migration, and tube formation in vitro and suppressed angiogenesis in vivo. Furthermore, AXIR siRNA induced cell arrest in the S/G2 phase while had no effect on cell apoptosis. We found that these subsequent effects might be via suppressing the expression of matrix metalloproteinase 2and matrix metalloproteinase 9. Conclusion: AXIR participates in angiogenesis, and may be a potential therapeutic target for angiogenesis related diseases.
\end{abstract}

\section{Introduction}

Angiogenesis is a process that new blood capillaries sprouting from pre-existing ones which is of great physiological and pathological importance [1]. It is a complicated process composed of multiple steps that include Endothelial cells (ECs) activation, guided sprouting proliferation, branching coordination, lumen formation, perfusion and vessel maturation [2].

H. Song and D. Pan contributed equally to this work.

Zhongtian Qi

and Shihong Zhao

KARGER 125
Department of Ophthalmology, Changhai Hospital, Second Military University,

Shanghai 200433 (China) and Department of Microbiology, Shanghai Key Laboratory of Medical Biodefense, Second Military University, Shanghai 200433 (China)

E-Mail zhaosh2001@sina.com, E-Mail qizt@smmu.edu.cn 
The excessive or insufficient angiogenic switch may lead to many kinds of diseases. Malignant, ocular and inflammatory disorders are the best-known pathological processes result from undue angiogenesis while ischemic heart disease, preeclampsia or vessel malformation are the most common disorders result from insufficient angiogenesis [3]. Therefore, it is of great significance to understand the molecular mechanisms of angiogenesis thoroughly which will shed new light on angiogenesis related disease therapy.

AXIIR, also known as Chromosome 5 open reading frame 39 (C5orf39), is originally cloned from human marrow cDNA, and identified as a receptor for Annexin II. AXIIR gene encodes a protein with 193 amino acids which is only detected in primate species up to now [4]. As a member of the Annexins family, Annexin Ilinvolves in many cell functions such as endocytic and exocytotic vesicular transport, interaction with cell adhesion molecules, regulation of ion channels, and being receptor for tenascin- $C$ and plasminogen [5-7]. Since AXIIR is originally identified as a receptor for Annexin II, several studies have investigated the role of AXIIR in mediating Annexin II signal. The researchers find that AXIIR can interact with Annexin IIto regulate cancer cell adhesion, migration and growth [8, 9]. However, some investigations suggest that AXIIR may not be the specific mediator of Annexin II signal [10]. It is noteworthy that AXIIR is found to be able to induce apoptosis in multiple human cell types and this property is Annexin II independent [11].These findings indicate that AXIIR may involve in cell function in a more broad way while not only mediate Annexin II signal.

In the present study, we investigated the role of AXIIRin regulation of angiogenic properties of HUVECs. We found AXIIR was expressed in HUVECs and knockdown of AXIIR expression using RNA interference technique could inhibit HUVECs proliferation, adhesion, migration and tube formation significantly. Furthermore, knockdown of AXIIR was able to suppress angiogenesis in nude mice. In order to better understand the molecular mechanism, we studied the affection of AXIIR siRNA on cell cycle, cell apoptosis and Matrix metalloproteinase 2 (MMP2), Matrix metalloproteinase 9 (MMP9) expression in HUVECs. We found AXIIRsiRNA could induce cell cycle arrest in the S/G2 phase while had no effect on cell apoptosis and could inhibit MMP2 and MMP9 expression significantly. The results obtained from this study demonstrate an important role of AXIIR in regulation of angiogenesis.

\section{Materials and Methods}

\section{Materials}

Fetal bovine serum, Trizol, Lipofectamine 2000and HRP-conjugated mouse and rabbit secondary antibodies were obtained from Invitrogen (Shanghai, China). Endothelial Cell Medium (ECM) was obtained from ScienCell (San Diego, USA). The anti-AXIIR and anti-CD31antibody were obtained from Santa Cruz Biotechnology Co., Ltd. (Dallas, USA). Antibodies to MMP2, MMP9 were obtained from Shanghai RuianBioTechnologies (Shanghai, China). The anti-AKT, anti-p-AKT, anti-ERK, anti-p-ERK, anti-TIMP2 antibodies were purchased from Bioworld Technology, Inc. (Nanjing, China). The anti-GAPDH antibody was obtained from Tianjin sungene Biotech (Tianjin, China). Matrigel Matrix was obtained from BD Biosciences (San Jose, USA). Cell-light EdU DNA cell proliferation kit was obtained from RiboBio (Guangzhou, China). PrimeScript RT reagents Kit, PrimeSTAR ${ }^{\circ}$ HS DNA Polymerase with GC Buffer and SYBR Premix Ex Taq were obtained from TAKARA BIOTECHNOLOGY (Dalian, China). Annexin V-FITC Apoptosis Detection Kit was obtained from eBioscience (San Diego, CA, USA). The transwell chamber and other cell culture plates were obtained from Corning Inc (NY, USA). All other regents were of analytical grade.

\section{Cell culture}

HUVECs were obtained from ScienCell (San Diego, USA) and cultured in Endothelial Cell Medium (ECM) supplemented with 10\% FBS, 1\% L-glutamine and were kept in a 5\% CO2 incubator at 100\% humidity and with the temperature of $37^{\circ} \mathrm{C}$.Passages 3 to 8 were used in all experiments.

Reverse transcription-PCR (RT-PCR)

Detection of AXIIR mRNA in HUVECs was performed using RT-PCR. Total RNA from HUVECs was extracted using Trizol reagent and then was reverse transcribed with PrimeScript 
RT reagents Kit according to the manufacture's protocol. Primer sequences for AXIIR were as follows $\left(5^{\prime} \rightarrow 3^{\prime}\right)$ : TCTAGAGCCGCCACCATGGAGCAACATTTTCTTGGC (forward) and GTCGACCTAAGGCTGCTTAGCTCCACAGATCCG (reverse). PCR reaction was performed using PrimeSTAR® HS DNA Polymerase with GC Buffer which gives the PCR product of 582bp. Finally, the products were analyzed by $2 \%$ agarose gel electrophoresis.

\section{RNA interference studies}

The siRNA was chemical synthesized by Shanghai GenePharma Co,Ltd. The company provided several sequences of negative control and we chose one that the composition of bases were highly similar to experimental group. The target sequence against AXIIR selected was sense CCACCUAUUGUGAGUUCAGTT and anti-sense CUGAACUCACAAUAGGUGGTT. The sequence of Negative control was sense UUCUCCGAACGUGUCACGUdTdT and anti-sense ACGUGACACGUUCGGAGAAdTdT. siRNA transfection was performed using Lipofectamine 2000 Reagent according to the manufacturer's instructions and the concentration used in the subsequent experiments was $50 \mathrm{nmol} / \mathrm{L}$.

\section{Cell proliferation assay}

Cell proliferation was determined by 5-ethynyl-2'-deoxyuridine (EdU) assay as previous described [12]. In brief, $5 \times 10^{3}$ cells were seeded in 96 -well plates and incubated overnight. Then, the cells were treated with AXIIRsiRNA or controls for $48 \mathrm{~h}$. We then dumped the medium and added $100 \mu \mathrm{l}$ fresh medium containing EdU $(100 \mu \mathrm{M})$. After incubation for 2 hours, the cells were stained as the following protocol: discard the EdU medium and fix the cells with $4 \%$ paraformaldehyde for 30 minutes. Then, wash with glycine for 5 minutes, followed by twice washes with $0.2 \%$ Trion X-100 of 10 minutes each time. Then, the cells were stained with Apollo fluorescent azide for 30 minutes, followed by three time washes of $0.2 \%$ Trion X-100. Furthermore, the cells were stained with Hoechst for 15 minutes at room temperature and washed with PBS for two times, and add $100 \mu \mathrm{l}$ PBS to further analysis. The images were taken and analyzed using a digital microscope system (IX81, Olympus).

\section{Cell migration assay}

For the cell migration assay, $1 \times 10^{5}$ HUVECs treated with AXIIR siRNA for $48 \mathrm{~h}$ were seeded in the upper chamber of transwell units with $8 \mu \mathrm{m}$ pore size polycarbonate filter in $0.5 \%$ FBS medium. The lower chamber was filled with $700 \mu \mathrm{L}$ ECM containing 1\% FBS. After incubation for 12 hours, the grown medium was removed and the filters were fixed with $4 \%$ paraformaldehyde for 20 minutes. Then, cells on the upper surface of the filter were completely removed by wiping with a cotton swab. Furthermore, the filters were stained with $0.1 \%$ Crystal violet for 15 minutes. The number of cells that migrated through the upper to the lower surface of the filter were counted and analyzed with a digital microscope system (IX81, Olympus).

\section{Cell adhesion assay}

After treatment with AXIIRsiRNA for $48 \mathrm{~h}, 5 \times 10^{3}$ HUVECs were harvested and seeded in a 96-well plate for $8 \mathrm{~h}$, unattached cells were rinsed gently with phosphate buffered saline (PBS). The remaining attached cells were fixed with $4 \%$ paraformaldehyde for 20 minutes. Then, the cells were stained with $0.1 \%$ Crystal violet for 15 minutes. Finally, the attached cells were counted and analyzed with a digital microscope system.

\section{Tube formation assay}

The tube formation assay was assessed following the protocols provided by the manufacturer. Briefly, $50 \mu \mathrm{l}$ Matrigel was added to 96 -well plate in each well and incubated at $37^{\circ} \mathrm{C}$ for 30 minutes for hardening. HUVECs treated with AXIIR siRNA for $48 \mathrm{~h}$ were harvested by trypsin and resuspended in $4 \times 10^{5}$ cells per $\mathrm{ml}$ with ECM. $100 \mu \mathrm{l}$ culture medium containing cells were gently added to the Matrigel-coated plates and incubated at $37^{\circ} \mathrm{C}$ for 4 hours. The capillary-like structures were then photographed with a digital microscope system.

In vivo Matrigel plug angiogenesis assay

HUVECs treated with AXIIRsiRNA for $12 \mathrm{~h}$ were harvested, and then a total of $1 \times 10^{7} \mathrm{HUVECs}$ in $50 \mu \mathrm{L}$ ECM mixed with $250 \mu \mathrm{L}$ Matrigel were subcutaneously injected into one site for four nude mice. Matrigel mixed with the cells polymerized to form a solid gel plug later. After inoculation for 7 days, the gel plugs 
was removed, fixed with formalin, embed in paraffin and section onto slides. The slides were stained with hematoxylin and eosin and anti-CD31 for immunohistochemistry. The CD31 positive staining was then photographed with a digital microscope system.

\section{Cell cycle assay}

Cultured HUVECs treated with AXIIRsiRNA for 48h were harvested using trypsin, washed in PBS, and fixed in $70 \%$ ethanol overnight. The fixed cells were then centrifuged to remove ethanol and washed with PBS for twice. HUVECs were resuspended in $0.5 \mathrm{ml}$ PBS containing $20 \mu \mathrm{g} / \mathrm{ml}$ propidium iodide and $200 \mu \mathrm{g} /$ $\mathrm{ml}$ RNAse and then incubated for 30 minutes at room temperature. The samples were analyzed using a MACSQuant Analyzer (MiltenyiBiotec, Teterow, Germany). The data were then analyzed using the Modfit software.

\section{Cell apoptosis assay}

The cell apoptosis assay was performed using Annexin V-FITC Apoptosis Detection Kit following the manufacturer' protocol. Cells treated with AXIIR siRNA for $48 \mathrm{~h}$ were harvested to perform the assay. The samples were analyzed using MACSQuant Analyzer and the data were analyzed by FLOWJO v7.6 software.

\section{Western blot analysis}

Total protein of HUVECs was extracted in a modified Buffer with 0.5\% SDS in the presence of proteinase inhibitor cocktail. Forty micrograms of proteins were electrophoresed in $12.5 \%$ SDS/PAGE mini gels and transferred onto poly vinylidenedifluoride membranes. The nonspecific sites were blocked with $5 \%$ nonfat milk before the membranes were incubated with primary antibodies at $4{ }^{\circ} \mathrm{C}$ overnight. Then, the blots were washed 3 times for 5 to 7 minutes each time in TBS-T. Blots were incubated with horseradish peroxidaseconjugated antibodies for 2 hours at room temperature, followed by 3 washes of 5 to 7 minutes each time in TBS-T. Finally, blots were incubated with Super Signal West Pico chemoluminescent substrate and visualized using the GeneGnome HR Image Capture System (Syngene, Frederick, MD, USA).

\section{Real-time quantitative PCR analysis}

Total RNA from HUVECs was extracted using Trizol reagent. Then, total RNA was reverse transcribed with PrimeScript RT reagents Kit. Equal quantities of cDNA were subjected to real-time PCR with using SYBR Premix Ex Taq while GAPDH was used as the control. Reactions were incubated at $95^{\circ} \mathrm{C}$ for 2 minutes, followed by 40 cycles at $95^{\circ} \mathrm{C}$ for 10 seconds, $55^{\circ} \mathrm{C}$ for 30 seconds and $72^{\circ} \mathrm{C}$ for 30 seconds. Reactions were run on Rotor Gene 3000A (Corbett Research, Australia) and the results were expressed as the mean relative value compared with control samples. The primers used are $\left(5^{\prime} \rightarrow 3^{\prime}\right)$ : GACACAGAAGCAGCAAGA (forward) and GCGAAGAATCCACTCCAG (reverse) forAXIIR, CAGCCAACTACGATGATGA (forward) and GTGCCAAGGTCAATGTCA (reverse) for MMP2 and AACCAATCTCACCGACAG (forward) and GGCAAGTCTTCCGAGTAG (reverse) for MMP9, TGGGCTACACTGAGCACCAG (forward) and AAGTGGTCGTTGAGGGCAAT (reverse) for GAPDH.

\section{Statistical analysis}

The data were analyzed using the GraphPad Prism 5 software, and the data were presented as the Means Standard Deviation (SD). One-way ANOVA and Tukey test were used for evaluate the data. A significance level of $\mathrm{p}<0.05$ was supposed to be statistically significant.

\section{Results}

\section{Expression of AXIIR in HUVECs and its role in cell proliferation}

There is no report on the expression of AXIIR in HUVECs and its role in angiogenesis. In this study, RT-PCR and Western blotting was used to detect the mRNA and protein expression of AXIIRin HUVECs (Fig. 1A, B). The AXIIRsiRNA could cause obvious reduction of AXIIR protein expression, where control groups showed no change (Fig. 1C). Cell proliferation assay showed that AXIIR siRNA could significantly inhibit the proliferation of HUVECs (Fig. 1D-E), which indicated that AXIIRwas essential in cell proliferation. 


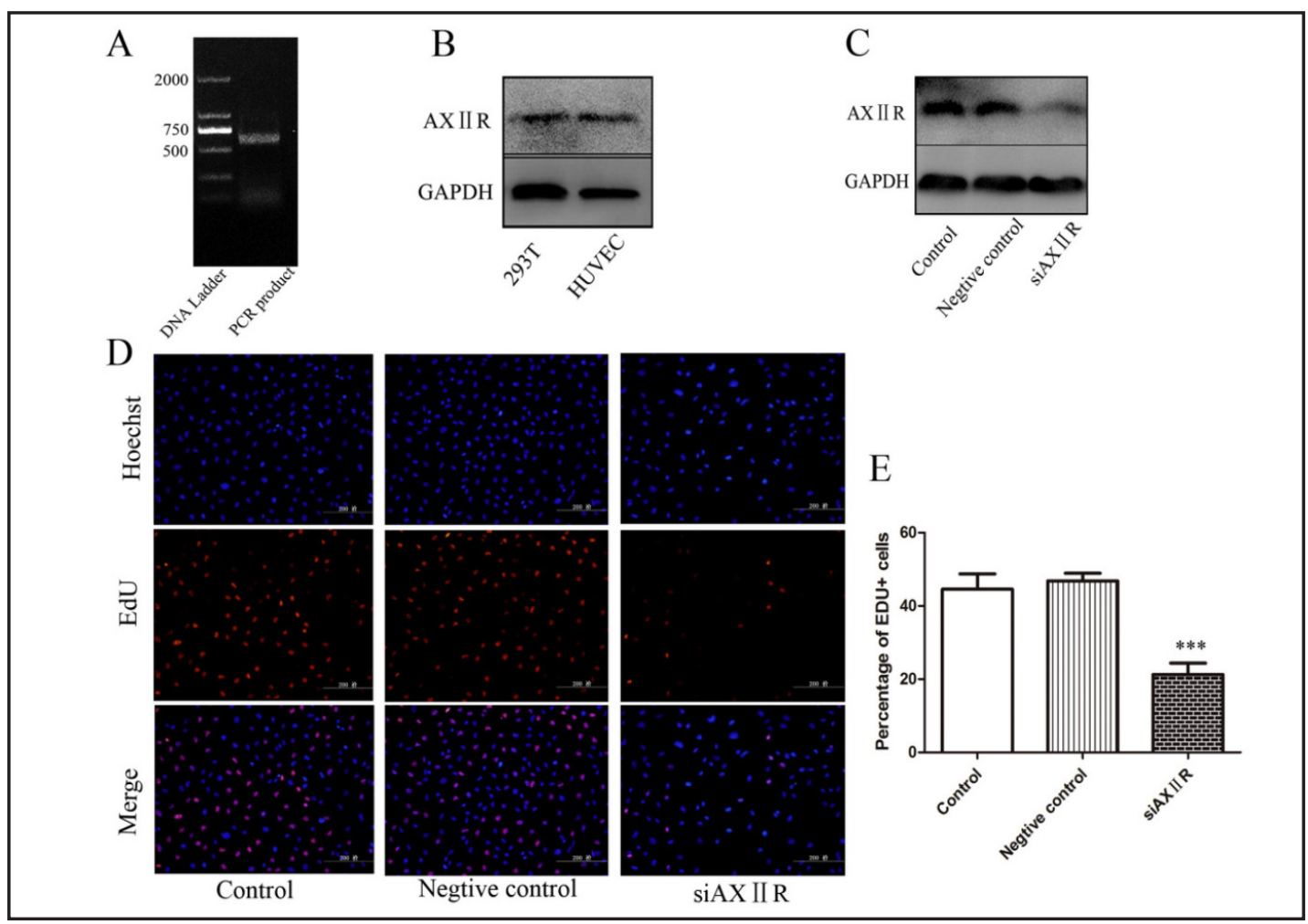

Fig. 1. AXIIR expresses in HUVECs and its role in cell proliferation. (A)Detection of AXIIR mRNAin HUVECs using RT-PCR. (B) Western blot analysis of AXIR expression in HUVECs using 293T cells as a positive control. (C) The transfection efficiency of AXIRsiRNA is determined by detecting AXIIR protein level. (D) Representative images of EdU-labeled replicating cells examined under a fluorescent microscope. (E) AXIIRsiRNA treated group shows a significant decrease in the cell proliferation ability $(n=3)$. Values are mean \pm SD of three separated experiments. Significant difference is observed compared with each group, ${ }^{* * *} p<0.001$.

\section{AXIIR siRNA inhibits cell migration and adhesion}

Cell migration is one of the key processes for endothelial cells to form new blood vessels in angiogenesis. We investigated whether knockdown of AXIIR would influence HUVECs migration ability. Treatment with AXIIR siRNA caused a significant decrease in the number of migrated cells compared to control groups (Fig. 2A, 2B). Since attachment of ECs to extracellular matrix is an important step that affects cell migration, we then examined the affection of treatment with AXIIRsiRNA on cell adhesion capability. We found that knockdown of AXIIR could significantly reduce the number of attached cells (Fig. 2C, Fig. 2D). Altogether, the data suggested that AXIIRwas required for ECs migration and adhesion.

\section{AXIIR siRNA inhibits angiogenesis in vitro and in vivo}

Another key step that involves in the process responsible for angiogenesis is tube formation of ECs. To examine the potential effects of AXIIR on endothelial cells tube formation, we studied how AXIIR affects HUVECs tube formation using two-dimensioned Matrigel assay. Compared with the control group, HUVECs treated with AXIIR siRNA showed a significant decrease in tube formation ability (Fig. 3A, B). In the in vivo Matrigel plug assay, knockdown of AXIIR was able to reduce anti-CD31 antibody-stained microvessel signals in nude mouse gel plugs (Fig. 3C, D). The result was consistent with tube formation assay in vitro, and suggested that AXIIRplayed a significant role in angiogenesis.

AXIIR siRNA induces cell cycle arrest while has no effect on cell apoptosis

Cell cycle is a complicated process happened in a cell which determines its division and duplication. The cell cycle plays an important role in cell growth as the cell must experience 


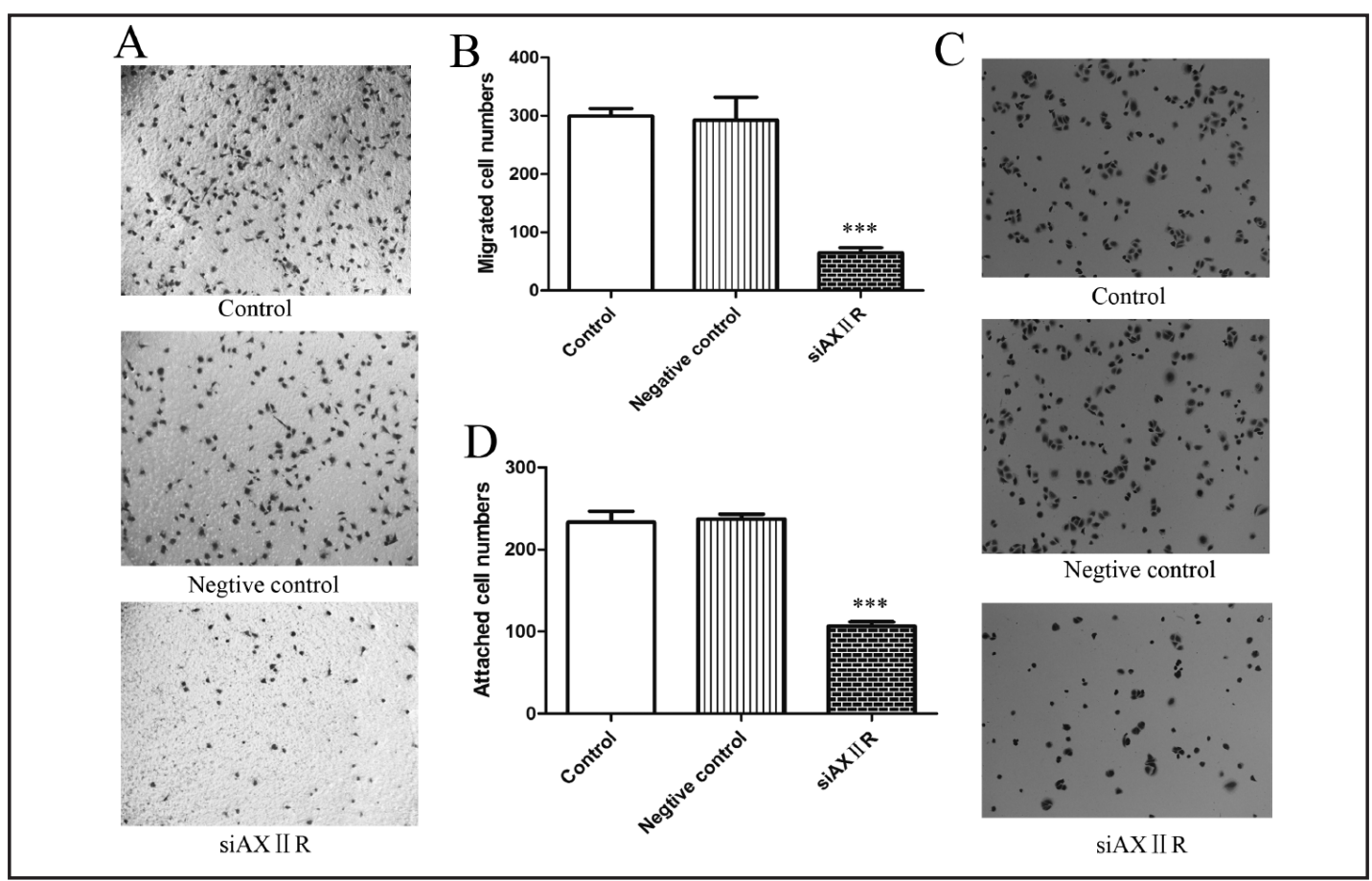

Fig. 2. AXIIRsiRNA inhibits HUVECs migration and adhesion. (A)Representative phase-contrast images of the migrated cells. (B) Migrated cells are quantified and presented as a histogram ( $n=3)$. (C) Representative phase-contrast images of the attached cells. (D) Attached cells are quantified and presented as a histogram $(\mathrm{n}=3)$. The data (mean \pm SD) are statistically significant where ${ }^{* * *} p<0.001$ of three separated experiments.

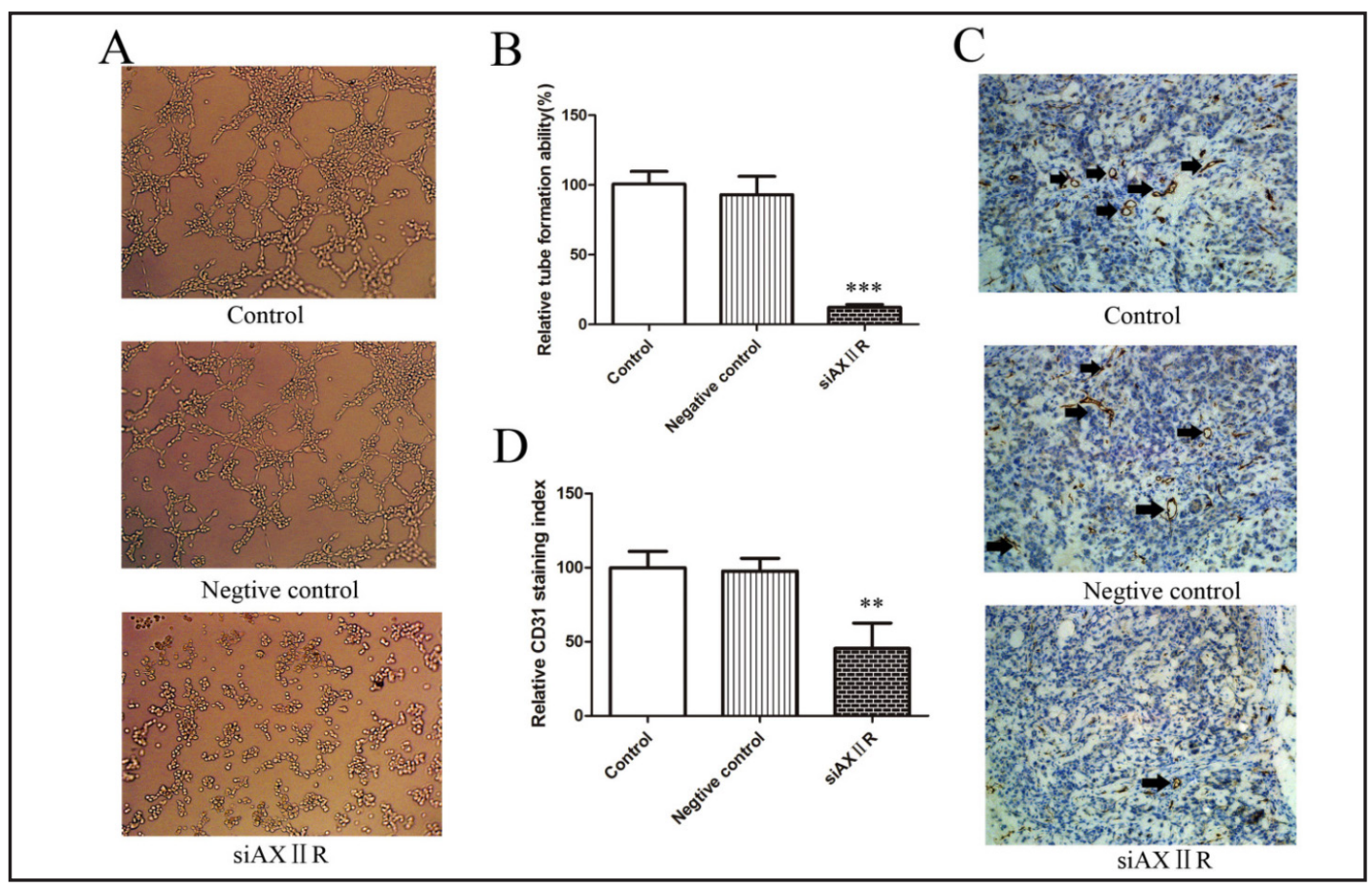

Fig. 3. AXIIRsiRNA inhibits angiogenesis in vitro and vivo. (A) Representative phase-contrast images of the tubes that formed. (B) The tube formation ability is quantified and presented as a histogram ( $n=3$ ). (C) Representative phase-contrast images of the in vivo Matrigel plug CD31 IHC staining (indicated by black arrows). (D) Summary of the relative blood vessel formation ability $(n=4)$. The data (mean + SD) are statistically significant of three separated experiments, ${ }^{* * *} p<0.001, * * p<0.01$. 


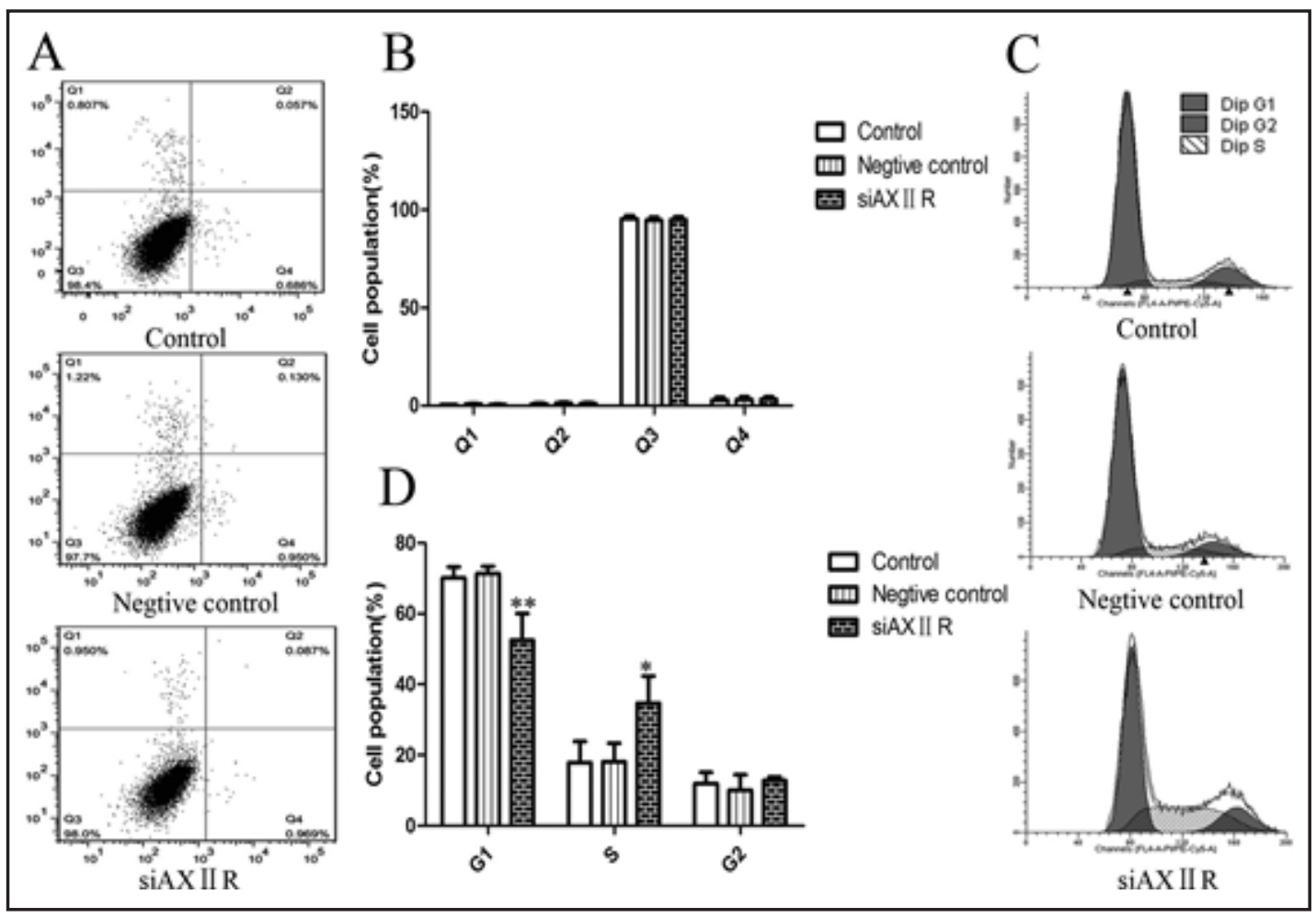

Fig. 4. AXIIRsiRNA induces cell cycle arrest while has no effect on apoptosis. (A) Representative images of the cell apoptosis analysis. Q1, Q2, Q3 and Q4 indicate the dead cells, apoptotic cells in late stage, live cells and apoptotic cells in early stage respectively. (B) The data of cell apoptosis assay are quantified and presented as a histogram which indicates that the cell apoptosis does not affect by AXIR siRNA. (C) Representative images of the cell cycle analysis. (D) The data of cell cycle assay are quantified and presented as a histogram. It shows that after treatment with AXIIRsiRNA there is an apparent increase in S phase and a decrease in G1 phase. The data (mean + SD) are statistically significant of three separated experiments, ${ }^{* *} p<0.01,{ }^{*} p<0.05$.

four different phases named G1 phase, S phase, G2 phase and M phase to proliferate [13]. Apoptosis is a process of programmed cell death by which the body could eliminate abnormal cells to maintain a better status [14].To determine whether AXIIR siRNA could change the cell cycle distribution and induce cell apoptosis, we performed flow cytometry at $48 \mathrm{~h}$ after siRNA interference. The results showed an increased cell population in the S-phase while no change in cell apoptosis after treatment with AXIIR siRNA (Fig. 4A, Fig. 4B, Fig. 4C, and Fig. 4D). The data indicated that AXIIRsiRNA exhibited an inhibitory effect on cell growth through induction of S-phase cycle arrest but not through induction of cell apoptosis of HUVECs.

\section{AXIIR siRNA suppresses pro-angiogenic factors expression to inhibit angiogenesis}

In addition, the molecular pathway in which AXIIRwas involved in regulating angiogenesis was determined by investigating the transcription level and protein level of angiogenesisrelated genes, MMP2 and MMP9. Our data showed that compared with control groups, HUVECs treated with AXIIRsiRNA exhibited a significant decrease in the expression of MMP2 and MMP9 both at mRNA level and protein level (Fig. 5A, Fig. 5B). Furthermore, AXIIR siRNA was able to increase the expression of tissue inhibitor of metalloproteinase 2 (TIMP2), which was regularly accompanied with the suppression of MMP2 and MMP9 (Fig. 5C) [15]. Many studies had indicated that ERK1/2 and AKT were involved in activities of MMP2 and MMP9 [16-18]. To assess whether the suppressed expression of MMP2 and MMP9 was mediated by the inhibition of AKT and ERK1/2 pathways, we investigated the effect of AXIIRsiRNA on phosphorylation of AKT and ERK1/2. We found that AXIIR siRNA was able to suppress the activation of AKT and ERK1/2 significantly (Fig. 5C). 


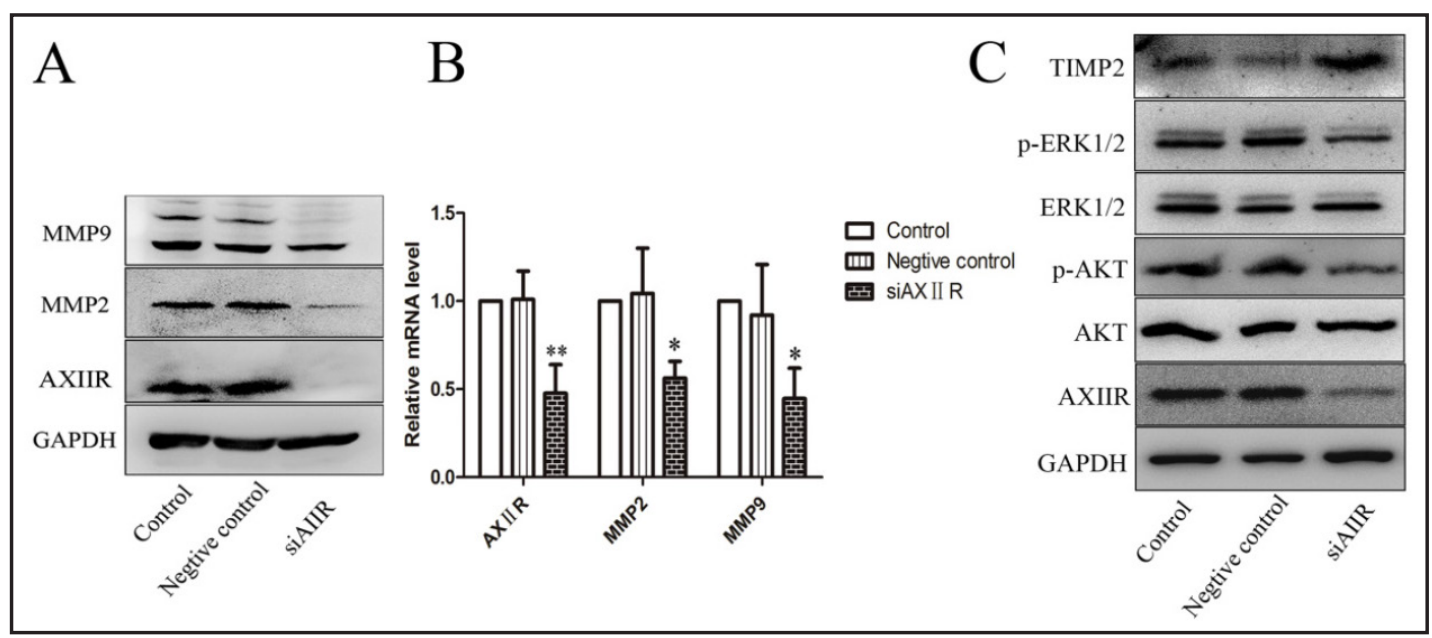

Fig. 5. Plausible mechanism of AXIR siRNA suppressed angiogenesis. (A) AXIRsiRNA is able to inhibit the expression of MMP2 and MMP9. (B) AXIIRsiRNA inhibits MMP2 and MMP9 mRNA expression. (C) AXIIRsiRNA suppressed the phosphorylation of AKT and ERK1/2, and increased the expression of TIMP2. HUVECs were grown in DMEM supplemented with 1\% FBS for 12 hours, and then cultured in DMEC supplemented with 10\% FBS for 30 minutes before analysis for phospho-S473-AKT, AKT, phosphor-T202/Y204-ERK1/2, ERK1/2. The data (mean \pm SD) are statistically significant of three separated experiments, ${ }^{*} p<0.05,{ }^{* *} p<$ 0.01 .

\section{Discussion}

Being critical to many disorders' occurrence and development, angiogenesis has drawn investigators' attention broadly. It has been reported that cell ingredients are able to regulate angiogenesis efficiently. The AXIIR, a newly identified protein, may be a promising one. We found for the first time that HUVECs express AXIIR and knockdown of AXIIR could significantly inhibit angiogenesis which might be through down-regulating the expression of MMP2 and MMP9.

AXIIR is expressed in most human cell types and mediates Annexin II signal by the activation of ERK1/2 and AKT pathways and induce apoptosis through the activation of Caspase-8 $[4,8,9,11]$. However, the expression of AXIRin HUVECs and its role in angiogenesis have not been investigated up to now. Different from the previous report that the protein level of AXIIRcould hardly detected by Western blotting and the mRNA was easily to detect, we found that both the protein and mRNA of AXIIR could be detected in HUVECs (Fig. 1A, B) [11]. The data suggest that HUVECs express AXIIR abundantly, and that the expression level and function of AXIIRmight vary in different cell types.

Angiogenesis is a complicated process that composed of multiple steps of which ECs differentiation, proliferation, migration, adhesion and tubulogenesis are of great importance $[1,19]$. The cell proliferation assay, cell migration assay, cell adhesion assay and tube formation assay are mature methods to imitate the angiogenesis process in vitro. The in vivo Matrigel plug assay is a mature method to imitate the angiogenesis process in vivo [2022]. Our data showed that AXIIRsiRNA was able to inhibit HUVECs proliferation, migration, adhesion, tube formation in vitro and inhibit angiogenesis in vivo (Fig. 1D, Fig. 2A, Fig. 2B, Fig. 3A, Fig. 3B). These results suggested a novel aspect for the role of AXIIRin HUVECs as a positive regulator of angiogenesis. The role of AXIIR in angiogenesis is consistent with that of Annexin II as we reported previously [23], which suggested that the function of AXIIR in angiogenesis might be Annexin II dependent. Yet, when over-expressing AXIIR in HUVECs using plasmid vector, we found the vector could induce apoptosis as well (unpublished data), which was Annexin II independent as reported [11]. Therefore, the relationship of Annexin II and AXIIR in angiogenesis remains indistinct which needs further investigation. 
To further reveal the inhibitory properties of AXIIR siRNA, cell apoptosis and cell cycle assay were performed using flow cytometry. Apoptosis is a process of programmed cell death by which the body was able to eliminating abnormal cells thus maintaining a well state [14, 24]. Although over-expression of AXIIR could induce cell apoptosis, our results demonstrated that knockdown of AXIIR had no effect on cell apoptosis (Fig. 4A, Fig. 4B). The cell cycle is a series of events leading to cell division and duplication. It plays a crucial role in regulating cell proliferation thus involving the angiogenesis process [24,25]. The data showed a remarkably increase of the percentage of cells of S phase(Fig. 4C, Fig. 4D).The result indicated that AXIR siRNA could induce cell arrest in the S/G2 phase where synthesis of DNA and centrosomes take place.

In order to better understand the molecular mechanism of AXIIR siRNA mediated suppression of angiogenesis, we investigated the pro-angiogenic factors AXIIR siRNA regulated. MMPs are a family of zinc-dependent endopeptidases which play important roles in various physiological and pathological processes [26, 27]. Of all the MMPs, MMP2 and MMP9 are the major ones involved in angiogenesis [28-31]. We found that AXIRsiRNA could significantly down-regulate MMP2 and MMP9 expression both at mRNA and protein level (Fig. 5A, Fig. 5B), accompanied with which was the increasing of TIMP2 (Fig. 5C). All of the results indicated an important role of AXIIR in degradation of the extracellular matrix. Furthermore, the subsequent effects might result from the inhibition of AKT and ERK pathways (Fig. 5C). Altogether, the results suggested that AXIIRsiRNA might be able to inhibit angiogenesis via inactivating phosphorylation of AKT and ERK1/2 thus reducing MMP2 and MMP9 expression.

In conclusion, we found that AXIIR was expressed in HUVECs and it played a vital role in angiogenesis. Using RNA interference technique to knockdown the expression of AXIIR in HUVECs, we demonstrated that AXIIR was essential in regulating cell proliferation, migration, invasion, tube formation in vitro and angiogenesis in vivo. Furthermore, we proved that knockdown of AXIIR could lead to cell cycle arrest in S/G2 phase while had no affection on cell apoptosis. Moreover, we found that the molecular mechanism might be the inhibition of expression of MMP2 and MMP9. All the data indicate that AXIIR may be a critical regulator in angiogenic responses of ECs.

\section{Disclosure Statement}

We declare no conflicts of interest.

\section{Acknowledgements}

This work was supported by the National Natural Science Foundation of China (No.81271017, No.81470652) and National Science and Technology Major Project (2011ZXJ9104-10C).

\section{References}

1 Potente M, Gerhardt H, Carmeliet P: Basic and therapeutic aspects of angiogenesis. Cell 2011;146:873-887.

-2 Adams RH, Alitalo K: Molecular regulation of angiogenesis and lymphangiogenesis. Nat Rev Mol Cell Biol 2007;8:464-478.

3 Carmeliet P: Angiogenesis in health and disease. Nat Med 2003;9:653-660.

4 Lu G, Maeda H, Reddy SV, Kurihara N, Leach R, Anderson JL, Roodman GD: Cloning and characterization of the annexin II receptor on human marrow stromal cells. J Biol Chem 2006;281:30542-30550.

5 Gerke V, Moss SE: Annexins: from structure to function. Physiol Rev 2002;82:331-371.

6 Flood EC, Hajjar KA: The annexin A2 system and vascular homeostasis. Vascul Pharmacol 2011;54:59-67.

7 Madureira PA, Surette AP, Phipps KD, Taboski MA, Miller VA, Waisman DM: The role of the annexin A2 heterotetramer in vascular fibrinolysis. Blood 2011;118:4789-4797.

8 Shiozawa Y, Havens AM, Jung Y, Ziegler AM, Pedersen EA, Wang J, Lu G, Roodman GD, Loberg RD, Pienta KJ, Taichman RS: Annexin II/annexin II receptor axis regulates adhesion, migration, homing, and growth of prostate cancer. J Cell Biochem 2008;105:370-380.

\section{KARGER}


-9 D'Souza S, Kurihara N, Shiozawa Y, Joseph J, Taichman R, Galson DL, Roodman GD: Annexin II interactions with the annexin II receptor enhance multiple myeloma cell adhesion and growth in the bone marrow microenvironment. Blood 2012;119:1888-1896.

10 Swisher JF, Burton N, Bacot SM, Vogel SN, Feldman GM: Annexin A2 tetramer activates human and murine macrophages through TLR4. Blood 2010;115:549-558.

11 Xiong Y, Fan C, Kong L, Dong L, Zhu N, Zhang J, Wang L, Qin T, Shen Y, Chen M: Annexin II receptor induces apoptosis independent of Annexin II. Apoptosis 2013;18:925-939.

12 Song H, Wang W, Zhao P, Qi Z, Zhao S: Cuprous oxide nanoparticles inhibit angiogenesis via down regulation of VEGFR2 expression. Nanoscale 2014;6:3206-3216.

13 Baumann K: Cell cycle: Making the spindle checkpoint strong. Nat Rev Mol Cell Biol 2014;15:430-430.

$\checkmark 14$ Brown JM, Attardi LD: The role of apoptosis in cancer development and treatment response. Nat Rev Cancer 2005;5:231-237.

15 Chen ML, Lin YH, Yang CM, Hu ML: Lycopene inhibits angiogenesis both in vitro and in vivo by inhibiting MMP-2/uPA system through VEGFR2-mediated PI3K-Akt and ERK/p38 signaling pathways. Mol Nutr Food Res 2012;56:889-899.

16 Fang JH, Zhou HC, Zeng C, Yang J, Liu Y, Huang X, Zhang JP, Guan XY, Zhuang SM: MicroRNA-29b suppresses tumor angiogenesis, invasion, and metastasis by regulating matrix metalloproteinase 2 expression. Hepatology 2011;54:1729-1740.

17 Babykutty S, PS P, RJ N, Kumar M, Nair MS, Srinivas P, Gopala S: Nimbolide retards tumor cell migration, invasion, and angiogenesis by downregulating MMP-2/9 expression via inhibiting ERK1/2 and reducing DNA-binding activity of NF-kB in colon cancer cells. Mol Carcinog 2012;51:475-490.

18 Xiao L-J, Lin P, Lin F, Liu X, Qin W, Zou H-F, Guo L, Liu W, Wang S-J, Yu X-G: ADAM17 targets MMP-2 and MMP-9 via EGFR-MEK-ERK pathway activation to promote prostate cancer cell invasion. Int J Oncol 2012;40:1714.

-19 Carmeliet P, Jain RK: Molecular mechanisms and clinical applications of angiogenesis. Nature 2011;473:298-307.

-20 Lo PH, Lung HL, Cheung AK, Apte SS, Chan KW, Kwong FM, Ko JM, Cheng Y, Law S, Srivastava G, Zabarovsky ER, Tsao SW, Tang JC, Stanbridge EJ, Lung ML: Extracellular protease ADAMTS9 suppresses esophageal and nasopharyngeal carcinoma tumor formation by inhibiting angiogenesis. Cancer Res 2010;70:5567-5576.

-21 Cheung AKL, Ko JM, Lung HL, Chan KW, Stanbridge EJ, Zabarovsky E, Tokino T, Kashima L, Suzuki T, Kwong DL-W: Cysteine-rich intestinal protein 2 (CRIP2) acts as a repressor of NF- $\mathrm{KB}-$ mediated proangiogenic cytokine transcription to suppress tumorigenesis and angiogenesis. Proc Natl Acad Sci U S A 2011;108:8390-8395.

22 Law EW, Cheung AK, Kashuba VI, Pavlova TV, Zabarovsky ER, Lung HL, Cheng Y, Chua D, Lai-Wan Kwong D, Tsao SW, Sasaki T, Stanbridge EJ, Lung ML: Anti-angiogenic and tumor-suppressive roles of candidate tumor-suppressor gene, Fibulin-2, in nasopharyngeal carcinoma. Oncogene 2012;31:728-738.

23 Zhao S-h, Pan D-y, Zhang Y, Wu J-h, Liu X, Xu Y: Annexin A2 promotes choroidal neovascularization by increasing vascular endothelial growth factor expression in a rat model of argon laser coagulation-induced choroidal neovascularization. Chin Med J 2010;123:713.

24 Evan GI, Vousden KH: Proliferation, cell cycle and apoptosis in cancer. Nature 2001;411:342-348.

25 Blagosklonny MV, Pardee AB: The restriction point of the cell cycle. Cell cycle 2002;1:102-104.

26 Chakraborti S, Mandal M, Das S, Mandal A, Chakraborti T: Regulation of matrix metalloproteinases: an overview. Mol Cell Biochem 2003;253:269-285.

27 Kessenbrock K, Plaks V, Werb Z: Matrix metalloproteinases: regulators of the tumor microenvironment. Cell 2010;141:52-67.

-28 Bergers G, Brekken R, McMahon G, Vu TH, Itoh T, Tamaki K, Tanzawa K, Thorpe P, Itohara S, Werb Z: Matrix metalloproteinase-9 triggers the angiogenic switch during carcinogenesis. Nat Cell Biol 2000;2:737-744.

29 Lambert V, Wielockx B, Munaut C, Galopin C, Jost M, Itoh T, Werb Z, Baker A, Libert C, Krell HW, Foidart JM, Noel A, Rakic JM: MMP-2 and MMP-9 synergize in promoting choroidal neovascularization. FASEB J 2003;17:2290-2292.

-30 Masson V, de la Ballina LR, Munaut C, Wielockx B, Jost M, Maillard C, Blacher S, Bajou K, Itoh T, Itohara S, Werb Z, Libert C, Foidart JM, Noel A: Contribution of host MMP-2 and MMP-9 to promote tumor vascularization and invasion of malignant keratinocytes. FASEB J 2005;19:234-236.

31 Adya R, Tan BK, Punn A, Chen J, Randeva HS: Visfatin induces human endothelial VEGF and MMP-2/9 production via MAPK and PI3K/Akt signalling pathways: novel insights into visfatin-induced angiogenesis. Cardiovasc Res 2008;78:356-365. 